\title{
Immunohistochemical and In Situ Hybridization Analyses of Midkine Expression in Thyroid Papillary Carcinoma
}

Masako Kato, M.D., Hiroyuki Maeta, M.D., Shinsuke Kato, M.D., Takao Shinozawa, Ph.D., Tadashi Terada, M.D.

Second Department of Pathology (MK, HM, TT) and Division of Neuropathology (SK), Faculty of Medicine, Tottori University, Yonago, Japan, Faculty of Engineering (TS), Gunma University, Kiryu, Japan.

Midkine (MK) is a novel heparin-binding growth factor whose gene has been identified in embryonal carcinoma cells in early stages of retinoic acidinduced differentiation. We immunohistochemically examined 90 thyroid papillary carcinomas (85 invasive type and five encapsulated type), using a rat IgG2a monoclonal antibody against the carboxyl terminal region of human MK in archival paraffin sections. The thyroid tumors exhibited an intense reaction in the cytoplasm. Most of the papillary carcinomas (77/90), had tumor cells that expressed MK. These were classified into the following two types: invasive type (76/85) and encapsulated type (1/5). Notably, the intensity of MK was stronger at the invading border area of the tumors than in the center. In tissues adjacent to the cancer tissues, normal follicular epithelial cells expressed MK very faintly or not at all. The in situ hybridization analysis revealed that the signals of MK transcripts were found in the cytoplasm of the cancer cells. In the noncancerous follicular epithelial cells adjacent to neoplasm the signals of MK transcripts were detected very weakly or not at all. The distribution and localization of the MK-transcript signals determined by in situ hybridization analysis were similar to those obtained by immunohistochemical analysis. We conclude that thyroid papillary carcinoma strongly expresses MK protein and messenger RNA, and that this overexpression may relate to the development and invasion of these carcinomas.

Copyright (C) 2000 by The United States and Canadian Academy of Pathology, Inc.

VOL. 13, NO. 10, P. 1060, 2000 Printed in the U.S.A.

Date of acceptance: April 20, 2000.

Address reprint requests to: Dr. Masako Kato, The Second Department of Pathology, Faculty of Medicine, Tottori University, 86 Nishi-machi, Yonago 683-8503, Japan; fax: 81-859-34-8348.
KEY WORDS: Immunohistochemistry, In situ hybridization, Midkine, Papillary carcinoma, Thyroid. Mod Pathol 2000;13(10):1060-1065

Midkine (MK), a novel heparin-binding growth factor found as a product of a retinoic acid-responsive gene, is a basic cysteine-rich polypeptide with a molecular weight of $15 \mathrm{kDa}$ (1-3). MK messenger RNA (mRNA) is strongly expressed in various tissues during the midgestation period of mouse embryogenesis $(4,5)$ and it is considered to be involved in regulation of organogenesis in mice $(6,7)$. Although mouse MK mRNA is expressed in the adult kidney, it decreases to undetectable levels with development in all other tissues.

Aberrant expression of growth factors is often associated with tumorigenesis, and its effects on the signal transduction system are considered to lead to tumorigenesis $(8,9)$. High expression of MK mRNA has been found in various human carcinomas such as Wilms' tumor; lung, breast, gastric, and colon carcinomas; and brain tumors (10-14). Other than brain tumors (14), there have been few immunohistochemical analyses of MK protein level in cancer tissues: only one rectal cancer (13) and 14 cases of esophageal cancer (15).

Furthermore, little is known of the expression of MK protein and mRNA levels in thyroid carcinomas. To clarify and characterize the production of MK protein by thyroid papillary carcinoma, the most frequent type of thyroid carcinoma, we immunohistochemically studied MK expression in the tumor tissues of 90 patients with papillary carcinomas using a new monoclonal antibody against the carboxyl terminal region of human-specific MK (14). Moreover, we studied MK mRNA transcript expression and its histological localization by in situ hybridization analysis in thyroid carcinomas.

In addition, colocalization between MK protein and MK mRNA in thyroid papillary carcinoma was examined using serial sections. 


\section{MATERIALS AND METHODS}

This retrospective study was carried out on surgical samples of 90 thyroid tumors ( 77 females and 13 males, aged 22 to 85 years). Formalin-fixed, paraffin-embedded cancer tissues were used. The thyroid tumors were classified according to the World Health Organization (WHO) International Histological Classification of Tumours (16). The 90 thyroid papillary carcinomas consisted of 85 cases of invasive type and five cases of encapsulated type. As normal controls, we used thyroid tissues that were anatomically far away from tumors in all 90 cases.

Multiple serial $4-\mu \mathrm{m}$ sections were prepared from each of the specimens. One section was stained with hematoxylin and eosin (H\&E) and the others were used for immunohistochemical and in situ hybridization analyses.

\section{Immunohistochemistry}

Monoclonal antibody against the carboxyl terminal region of human MK (concentration: $2 \mu \mathrm{g} / \mathrm{mL}$ ) was used (14). The avidin-biotin-immunoperoxidase complex (ABC) method was employed for MK detection (17). Sections were deparaffinized, endogenous peroxidase activity was quenched by incubation for 30 min with $0.3 \% \mathrm{H}_{2} \mathrm{O}_{2}$, and then washed with phosphate-buffered saline (PBS). Normal serum homologous with the secondary antibody served as the blocking reagent. Tissue sections were incubated with the monoclonal antibody for $18 \mathrm{~h}$ at $4^{\circ} \mathrm{C}$; sections exposed to PBS and nonimmunized rat IgG served as controls. Regarding the preabsorption test, some sections were incubated with anti-MK antibody that had been preabsorbed with an excess of the synthetic human MK antigen. Bound antibodies were visualized by the $A B C$ method using the appropriate Vectastain $\mathrm{ABC}$ kit (Vector Laboratories, Burlingame, $\mathrm{CA}$ ) and 3,3'-diaminobenzidine tetrahydrochloride as the chromogen. In some sections, methyl green was used as counterstain. MK immunoreaction was considered positive when the cell cytoplasm was stained, irrespective of the percentage of positive cells.

\section{In Situ Hybridization}

Three antisense oligonucleotides against exons 1, 2, and 5 of human MK DNA were synthesized by a DNA automatic synthesizer (exon 1, 5'-GGGCCCGGCGCGGGAGGGAGCGAAGCAGCGCGGGCAGCGAGCGA-3'; exon 2, 5' -ATGCAGCACCGAGGCTTCCT-3'; exon 5, 5'-ACTAGACGCCAAGCCTGGAT- $\left.3^{\prime}\right)$. These probes were mixed and used for in situ hybridization.

Oligonucleotide sense probes were also synthesized, and they were mixed and used as negative controls. RNase treatment of sections was per- formed before hybridization as another negative control. They were labeled with digoxigenin (DIG) according to the DIG3'-end-labeling method using a DIG-Oligonucleotide Tailing kit (Boehringer Mannheim, Mannheim, Germany).

Details of the in situ hybridization technique have been described previously (14). The in situ hybridization was performed on all thyroid tumors. The tissue sections, which were prepared under sterile conditions, were deparaffinized and washed with PBS.

They were washed twice with $2 \mathrm{mg} / \mathrm{mL}$ of glycine in PBS, acetylated for $20 \mathrm{~min}$, and then washed in SSC (sodium chloride-sodium citrate solution). After prehybridization, tissue sections were incubated with either antisense or sense probes of $\mathrm{MK}$ for $24 \mathrm{~h}$ at $42^{\circ} \mathrm{C}$ in an incubation chamber. They were washed in SSC solution. To detect the hybridization MK transcripts, we used a DIG-Nucleic Detection kit (Boehringer Mannheim). Briefly, tissue sections were washed with DIG-buffer 1 (100 mm Tris-HCl pH 7.5, 20 ${ }^{\circ}$ C, $150 \mathrm{~mm}$ sodium chloride). After blocking, they were incubated with alkaline phosphatase-containing nitroblue tetrazolium salt and levamisole in DIG-buffer $3(100 \mathrm{~mm}$ Tris- $\mathrm{HCl}$ (pH 9.5), $100 \mathrm{~mm} \mathrm{NaCl}, 50 \mathrm{~mm} \mathrm{MgCl}_{2}$ ).

The color reaction was developed for 12 to $40 \mathrm{~h}$ in a dark room and then stopped by incubating the sections in DIG-buffer 4 (10 mM Tris- $\mathrm{HCl}$ [pH 8.0], 1 mM EDTA).

Statistical analysis was performed by the Fisher exact test.

\section{RESULTS}

Histologically, the majority of papillary carcinoma cells exhibited the papillary growth pattern (Fig. 1A). The cancer cells had ground glass nuclei with inconspicuous nucleoli. Nuclear pseudoinclusions and nuclear grooves were apparent in some specimens (Fig 1A). Tissue sections were classified into invasive papillary carcinomas (85 cases) and encapsulated type (five cases) (Fig. 2A).

The results are summarized in Table 1 . When control sections were incubated with PBS and nonimmunized rat IgG, immunoreaction products were not detected. In addition, the anti-MK antibody, pretreated with an excess of the synthetic human MK antigen, did not stain sections with any immunoreaction products.

Although the proportion of positively stained versus unstained tumor cells varied among samples to sample, MK-positive specimens comprised more than $80 \%$ of cancer cells that expressed MK. Cytoplasmic staining with MK antibody was observed in many tumors, and the staining patterns were heterogenous (Fig. 1B). No immunoreactivity was found in vascular or mesenchymal cells among all 

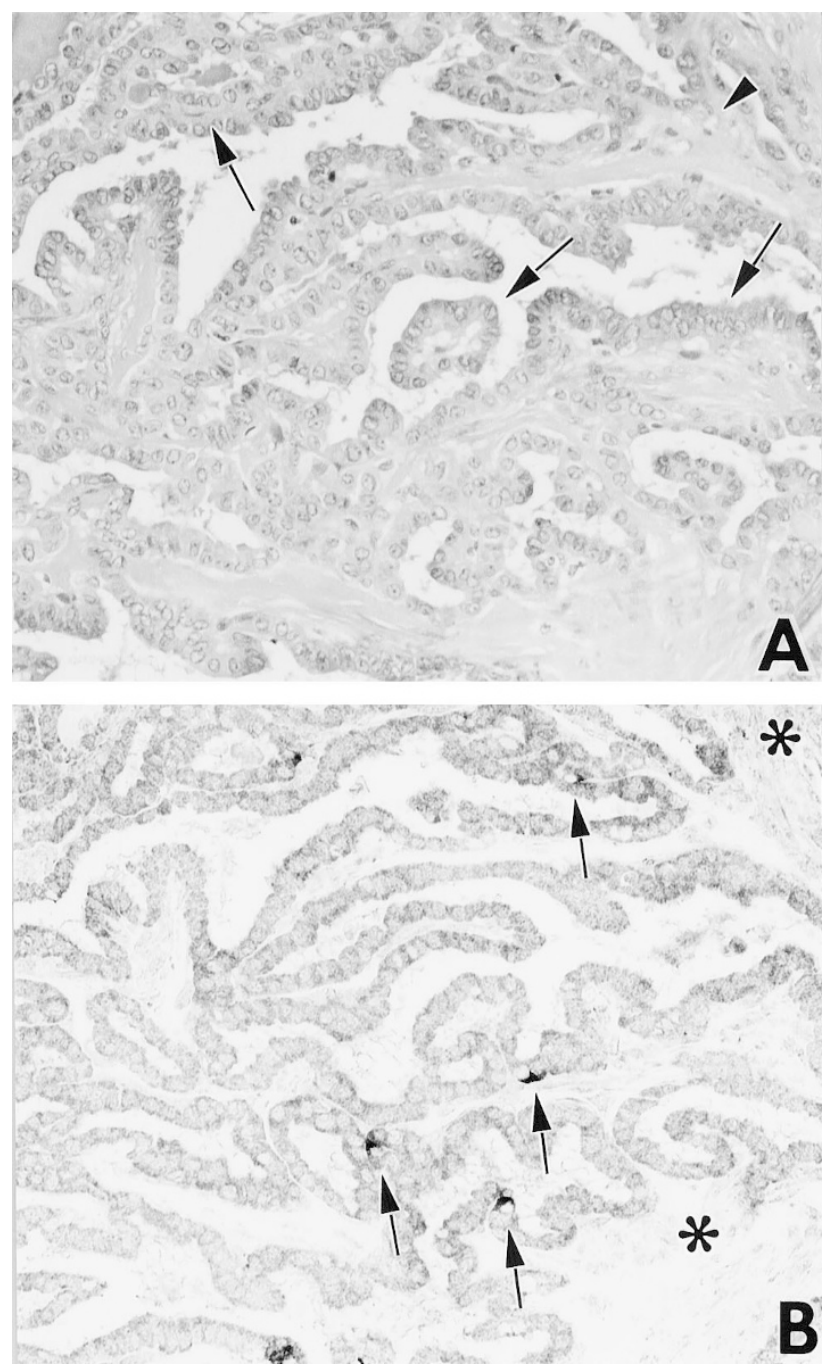

FIGURE 1. Serial sections of invasive papillary carcinoma in thyroid stained with hematoxylin and eosin (A) and immunostained by the rat IgG2a monoclonal antibody against human specific MK (B). A, this tumor displays a papillary pattern. Cancer cell has a ground glass nucleus with a nuclear groove (arrows). Capillaries in stromal tissues can be seen (arrowhead) (original magnification, $230 \times$ ). B, the cytoplasm of cancer cells is positively stained with anti-MK antibody. Some cells are stained intensely (arrows). Capillaries and mesenchymal tissues (asterisks) were not stained (no counterstain; original magnification, $230 \times$ ).

cancers examined. A positive reaction with anti-MK antibody was observed in 77 of the 90 thyroid papillary carcinomas $(85 \%)$.

Seventy-six of 85 invasive papillary carcinomas $(89 \%)$ expressed MK protein. In contrast, in the encapsulated variant, four of five specimens exhibited tumor cells that expressed MK very faintly or not at all (Fig. 2B). The normal thyroid tissues adjacent to cancer tissues were not immunostained by the anti-MK antibody. (Fig. 2B). In the invasive type, the percentage of MK-positive cases was significantly higher than that of MK-positive cases in encapsulated types (Fisher exact test, $P=.0012$ ). It is of interest that in invasive cases, the intensity of MK expression was stronger at the invading border
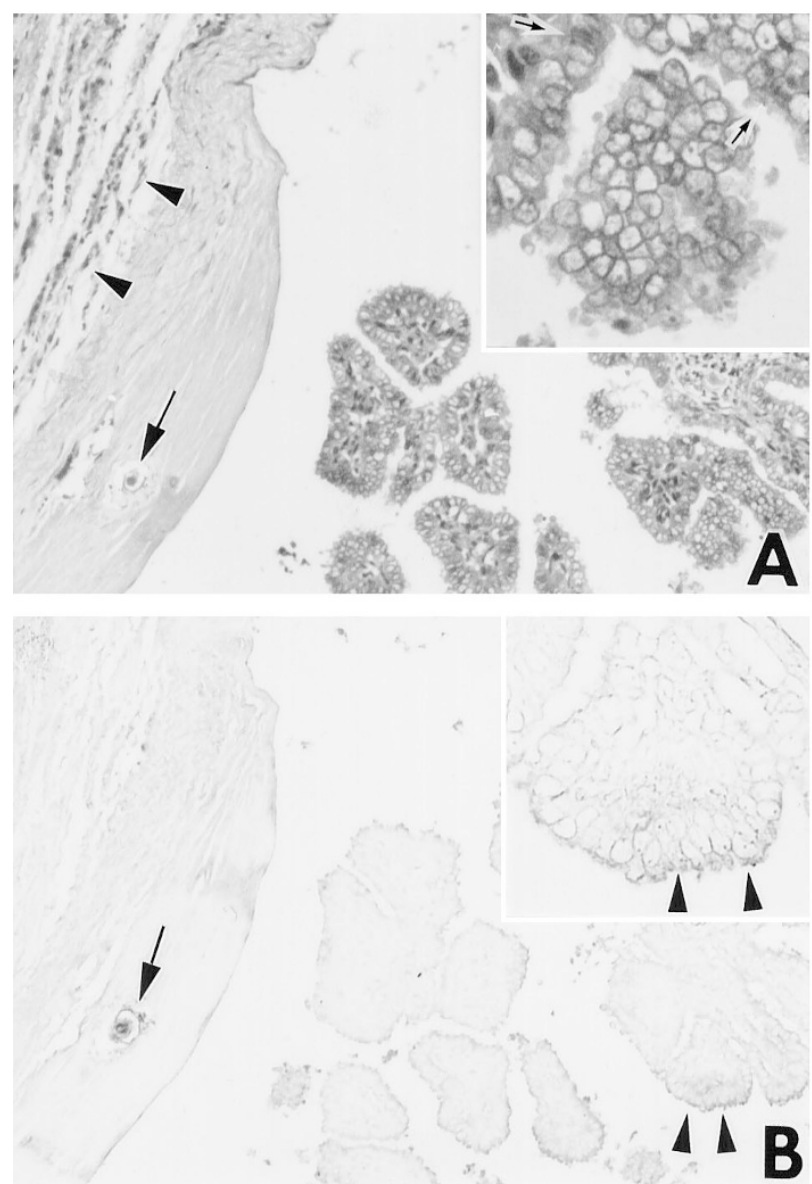

FIGURE 2. Serial sections of encapsulated papillary carcinomas stained with hematoxylin and eosin (A) and immunostained anti-MK antibody (B). A, papillary tumor is encapsulated by thick connective tissue. The surrounding thyroid tissues are compressed and atrophic (arrowheads). A psammoma body is found in the thick capsular tissues (arrow) (original magnification, 140×). Inset, nuclear grooves are present in some cancer cells (arrows) (original magnification, 500×). B, almost all of the cancer cells were unstained by anti-MK antibody; however, a few cancer cells expressed MK very faintly (arrowheads). Thick fibrous capsules, including noncancerous normal follicular epithelial cells, are negative for MK. A calcified psammoma body can be seen (arrow) (no counterstain; original magnification, 140×). Inset, some cancer cells expressed MK very faintly (arrowheads) (original magnification, $400 \times)$.

TABLE 1. Immunohistochemical Expression of Midkine in Thyroid Papillary Carcinoma

\begin{tabular}{crc}
\hline & No. of Cases & MK-Positive Cases (\%) \\
\hline Papillary carcinoma & 90 & $77(85)$ \\
Invasive type & 85 & $76(89)^{a}$ \\
Encapsulated type & 5 & $1(20)^{b}$
\end{tabular}

There is a significant difference between ${ }^{a}$ and ${ }^{b}$ (Fisher exact test, $P=$ .0012).

of the tumor than in the center of the tumor tissues (Fig. 3, A-B). Vascularization was observed near the invading cancer cells that exhibited intense $\mathrm{MK}$ staining (Fig. 3, A-B). There were no significant differences in MK expression between age and gender in papillary thyroid carcinoma.

The analysis of 90 thyroid papillary carcinomas using antisense probes revealed the presence of 

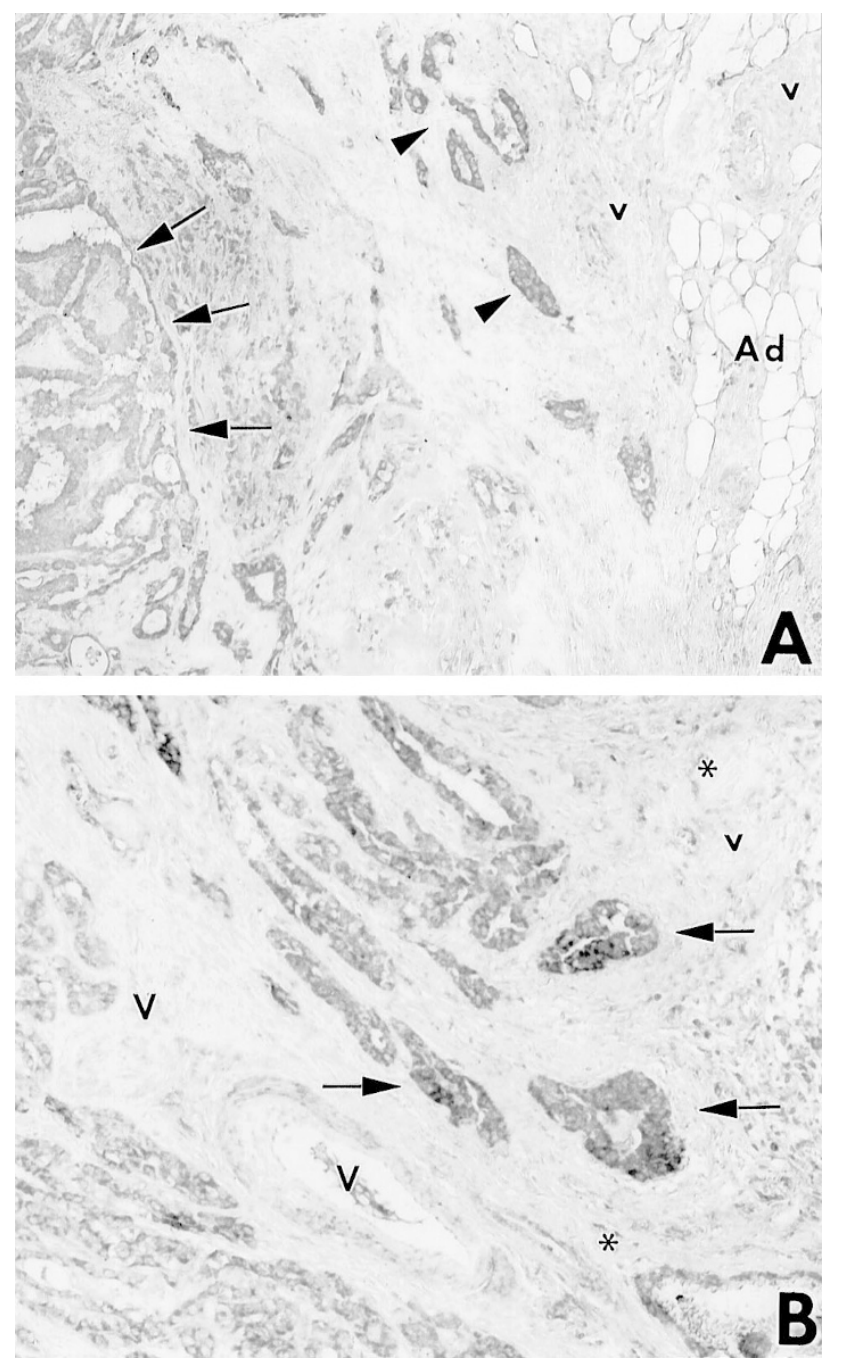

FIGURE 3. MK immunostaining of invading papillary carcinomas. A, invading cancer nests (arrowheads) express MK stronger than central cancer tissues (arrows). No significant immunoreactivities are found in vascular (V) and mesenchymal tissues. The surrounding adipose tissues (Ad) are negative for MK (no counterstain; original magnification, $90 \times$ ). B, cancer cells in border area express MK protein intensely (arrows). Small vessels (V) and mesenchymal tissues (asterisks) are negative for MK (counterstain for methyl green; original magnification, 140×).

specific signals in the tumor cell cytoplasm (Fig. $4 \mathrm{~A})$. In control sections, no signals were detected with RNase pretreatment. Noncancerous follicular epithelial cells adjacent to neoplasm expressed MK mRNA very weakly or not at all. There was a direct correspondence between in situ hybridization and the immunohistochemically determined levels of MK expression among the 90 papillary carcinomas (Figs. 1B and 4A). Hybridization with the sense probe revealed no labeling of these tumor cells (Fig. $4 \mathrm{~B})$.

\section{DISCUSSION}

In the mouse embryo, the MK mRNA was first detected on the seventh day of gestation using in situ hybridization analysis (5).
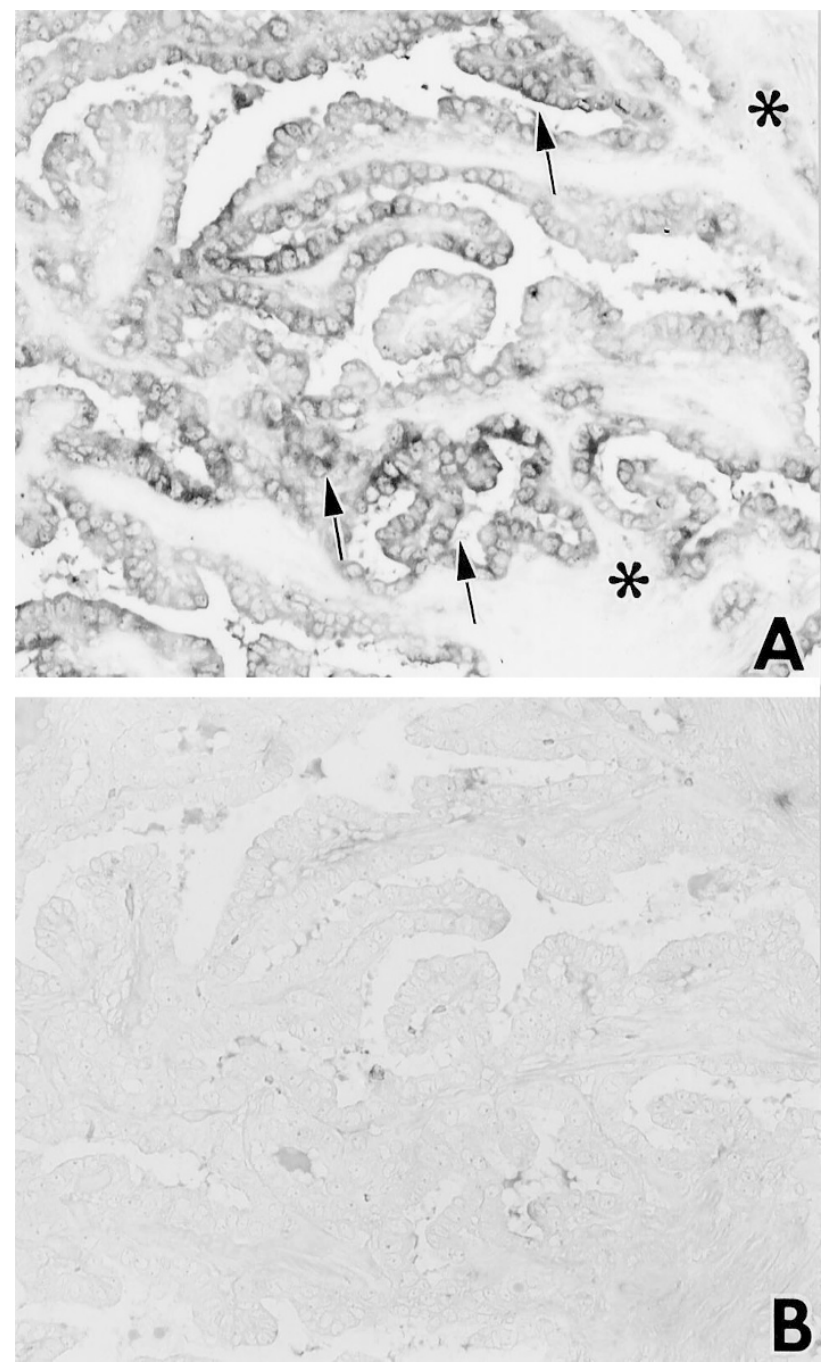

FIGURE 4. The serial sections consecutive to Figure 1, A-B of the expression of MK transcripts detected by in situ hybridization in thyroid papillary carcinoma. A, in situ hybridization with antisense probe. Demonstration of MK transcripts in papillary carcinoma shown by the intense labeling of the cytoplasm in some cancer cells. The cancer cells labeled intensely by in situ hybridization analysis (arrows) almost coincide with the cells that strongly expressed MK protein by immunohistochemistry (arrows in Fig. 1B). No significant signals are detected in mesenchymal components (asterisks) (original magnification, $230 \times$ ). B, in situ hybridization with sense probe of the section consecutive to A. No significant signals were detected in this section (original magnification, $230 \times$ ).

MK mRNA expression is quite strong in some organs, such as the brain, stomach, lung, and small intestine, from the 11th to 13th days of gestation (4, 5). In adult mice, only the kidney continues to express MK (3-5). We cannot readily compare animal tissues to human tissues; we failed to detect the significant signals of MK transcripts in thyroid noncancerous normal tissues of humans aged 22 to 85 .

Our novel finding was that MK mRNA was overexpressed in human papillary carcinoma of thyroid. Strong expression of MK mRNA has been reported in various human carcinomas, such as gastric, colon, breast, and lung cancer, and brain tumors (1014). In gastric and colorectal carcinomas, the MK 
mRNA level was higher in cancer specimens than in the corresponding noncancerous tissues (13). Although normal breast and lung tissues did not express MK mRNA, it was frequently expressed in malignant breast and lung tissues $(11,12)$. In our recent study of brain tumors, glioblastomas expressed MK mRNA strongly, although normal brain tissues did not (14). Breast and lung carcinomas, as well as glioblastomas, have different origins. However, these malignant tumor tissues overexpressed MK mRNA, whereas the corresponding noncancerous normal tissues did not $(11,12,14)$. Our results regarding MK mRNA overexpression in thyroid papillary carcinomas coincide with those of the carcinomas originated from the different organs mentioned above.

As for immunohistochemical analyses of MK expression in human cancer tissues, only two other studies on colorectal and esophageal carcinomas using immunohistochemical methods have been reported previously: the former examined one case of rectal cancer (13), the latter 14 esophageal cancers (15). In addition, the antibody used in the studies of the colorectal (13) and esophageal cancers (15) was a polyclonal antibody against mouse MK, not anti-human MK antibody. In our recent immunohistochemical analysis of brain tumors, we used a monoclonal antibody against the carboxyl terminal region of human-specific MK (14). We applied this monoclonal antibody to paraffin sections and revealed intensely positive staining for MK in the tumor cytoplasm (14). In this study, we used this antibody in thyroid papillary carcinomas and detect intensely positive staining for $\mathrm{MK}$ in the tumor cytoplasm. Approximately $85 \%$ of the cases of papillary carcinoma exhibited tumor cells that expressed MK. The distribution and localization of the MK protein determined by immunohistochemistry were similar to those obtained by in situ hybridization analysis.

The Wilms' tumor cell line G401 and PA-1 teratocarcinoma cells (10), as well as fetal human astrocytes (18), secrete MK protein into the culture medium. Moreover, MK exhibits potent trophic activity towards neurons (18-20). Although the molecular receptor for MK has not yet been identified $(21,22)$, fetal astrocytic MK secretion is thought to occur through a paracrine mechanism. In mouse embryos, MK is suspected to have a paracrine function at midgestational development stages (7). On the other hand, it has been suggested that the overexpression of $\mathrm{MK}$ is related to oncogenesis of Wilms' tumors (10) and glioblastomas (14) in an autocrine manner. In malignant tumors, the endogenous production of growth factors by tumor cells, which in turn act on themselves via receptors, may lead to progression of malignancy in an autocrine manner (23). In thyroid papillary carcinomas, it has been suggested that transforming growth factor alpha and epidermal-growth-factor receptor are involved in an autocrine loop (24). Considered in connection with the fact that MK is a member of a novel family of heparin-binding growth factors (13 ), the aberrant MK expression in thyroid papillary carcinomas might reflect the amplification of autocrine activity.

The intense MK-expression of tumor cytoplasm observed in invading border areas was accompanied by small vessels. Although the ultimate biological significance has not been established, a role as an angiogenic factor of $\mathrm{MK}$ is suspected in bladder cancer (25). Together with our findings of vascularization near the intensely MK-stained cancer cells in invading areas, more abundant blood supply, based on angiogenic activity in invading border areas than in the center, might be related to the intense MK expression in border areas of thyroid papillary carcinomas.

Our results suggest that increased MK expression may play a role in the development and invasion of thyroid papillary carcinoma. Whether these considerations apply to thyroid papillary carcinoma without the benefit of normal control mechanisms remains to be determined.

\section{REFERENCES}

1. Kadomatsu K, Tomomura M, Muramatsu T. cDNA cloning and sequencing of a new gene intensity expressed in early differentiation stages of embryonal carcinoma cells and in mid-gestation period of mouse embryogenesis. Biochem Biophys Res Commun 1988;151:1312-8.

2. Tomomura M, Kadomatsu K, Matsubara S, Muramatsu T. A retinoic acid-responsive gene, $\mathrm{MK}$, found in the teratocarcinoma. J Biol Chem 1990;265:10765-70.

3. Muramatsu T. Midkine (MK), the product of a retinoic acid responsive gene, and pleiotrophin constitute a new protein family regulating growth and differentiation. Int J Dev Biol 1993;37:183-8.

4. Muramatsu H, Shirahama H, Yonezawa S, Maruta H, Muramatsu T. Midkine, a retinoic acid-inducible growth/differentiation factor: immunochemical evidence for the function and distribution. Dev Biol 1993;159:392-402.

5. Kadomatsu K, Huang RP, Suganuma T, Murata F, Muramatsu T. A retinoic acid responsive gene MK found in the teratocarcinoma system is expressed in spatially and temporally controlled manner during mouse embryogenesis. J Cell Biol 1900;110:607-16.

6. Mitsiadis TA, Salmivirta M, Muramatsu T, Muramatsu H, Rauvala H, Lehtonen E, et al. Expression of the heparinbinding cytokines, midkine (MK) and HB-GAM (pleiotrophin) is associated with epithelial-mesenchymal interactions during fetal development and organogenesis. Development 1995;121:37-51.

7. Mitsiadis TA, Muramatsu T, Muramatsu H, Thesleff I. Midkine (MK), a heparin-binding growth/differentiation factor, is regulated by retinoic acid and epithelial-mesenchymal interactions in the developing mouse tooth, and affects cell proliferation and morphogenesis. J Cell Biol 1995;129:267-81.

8. Aaronson SA. Growth factors and cancer. Science 1991;254: 1146-53. 
9. Cross M, Dexter TM. Growth factors in development, transformation, and tumorigenesis. Cell 1991;64:271-80.

10. Tsutsui J, Kadomatsu K, Matsubara S, Nakagawara A, Hamanoue M, Takao S, et al. A new family of heparin-binding growth/differentiation factors: Increased midkine expression in Wilms' tumor and other human carcinomas. Cancer Res 1993;53:1281-5.

11. Garver RI, Chan CS, Milner PG. Reciprocal expression of pleiotrophin and midkine in normal versus malignant lung tissues. Am J Respir Cell Mol Biol 1993;9:463-6.

12. Garver RI, Radford DM, Donis-Keller H, Wick MR, Milner PG. Midkine and pleiotrophin expression in normal and malignant breast tissue. Cancer 1994;74:1584-90.

13. Aridome K, Tsutsui J, Takao S, Kadomatsu K, Ozawa M, Aikou $\mathrm{T}$, et al. Increased midkine gene expression in human gastrointestinal cancers. Jpn J Cancer Res 1995;86:655-61.

14. Kato S, Ishihara K, Shinozawa T, Yamaguchi H, Asano Y, Saito M, et al. Monoclonal antibody to human midkine reveals increased midkine expression in human brain tumors. J Neuropathol Exp Neurol 1999;58:430-41.

15. Miyauchi M, Shimada H, Kadomatsu K, Muramatsu T, Matsubara S, Ikematsu S, et al. Frequent expression of midkine gene in esophageal cancer suggests a potential usage of its promoter for suicide gene therapy. Jpn J Cancer Res 1999;90: 469-75.

16. Hedinger, C, Williams, ED, Sobin LH, editors. Histological Typing of Thyroid Tumours. 2nd ed. International Histological Classification of Tumours. World Health Organization. Berlin: Springer-Verlag; 1988.

17. Hsu SM, Raine L, Fanger H. Use of avidin-biotin-peroxidase complex $(\mathrm{ABC})$ in immunoperoxidase techniques: a compar- ison between $\mathrm{ABC}$ and unlabeled antibody (PAP) procedures. J Histochem Cytochem 1981;29:577-80.

18. Satoh J, Muramatsu H, Moretto G, Muramatsu T, Chang HJ, Kim ST, et al. Midkine that promotes survival of fetal human neurons is produced by fetal human astrocytes in culture. Dev Brain Res 1993;75:201-5.

19. Michikawa M, Kikuchi S, Muramatsu H, Muramatsu T, Kim SU. Retinoic acid responsive gene product, midkine, has neurotrophic functions for mouse spinal cord and dorsal root ganglion neurons in culture. J Neurosci Res 1993;35: $530-9$.

20. Kikuchi S, Muramatsu H, Muramatsu T, Kim SU. Midkine, a novel neurotrophic factor, promotes survival of mesencephalic neurons in culture. Neurosci Lett 1993;160:9-12.

21. Matsubara S, Take M, Pedraza C, Muramatsu T. Mapping and characterization of a retinoic acid-responsive enhancer of midkine, a novel heparin-binding growth/differentiation factor with neurotrophic activity. J Biochem 1994;115:108896.

22. Tomomura M, Kadomatsu K, Nakamoto M, Muramatsu H, Kondoh $\mathrm{H}$, Imagawa $\mathrm{K}$, et al. A retinoic acid responsive gene, MK, produces a secreted protein with heparin binding activity. Biochem Biophys Res Commun 1990;171:603-9.

23. Sporn MB, Roberts AB. Autocrine growth factors and cancer. Nature 1985;313:745-7.

24. Haugen DRF, Akslen LA, Varhaug JE, Lillehaug JR. Demonstration of a TGF-alpha-EGF receptor autocrine loop and c-myc protein over expression in papillary thyroid carcinomas. Int J Cancer 1993;55:37-43.

25. O'Brien T, Cranston D, Fuggle S, Bicknell R, Harris AL. The angiogenic factor midkine is expressed in bladder cancer, and overexpression correlates with a poor outcome in patients with invasive cancers. Cancer Res 1996;56:2515-8. 